Edición Extraordinaria. p.p. $129-138$

Memorias del VIII Encuentro Nacional de Experiencias en Enseñanza de la Biología y la Educación Ambiental. III Congreso Nacional de Investigación en Enseñanza de la Biología.

\title{
ESTUDIO DE LA IMAGEN DE CIENCIA EN ESTUDIANTES DE SECUNDARIA A TRAVÉS DE SU INTERACCIÓN CON UNA ESTRATEGIA DIDÁCTICA MEDIADA POR UN VIDEOJUEGO, EN TORNO AL CONCEPTO DE HUMEDAL.
}

\author{
Abella Susana ${ }^{1}$
}

\section{RESUMEN}

La naturaleza de las ciencias (NOS) es una metaciencia que articula la epistemología, la historia y la sociología de las ciencias con el fin de contribuir a la reflexión sobre la imagen de ciencia desde cuestiones sociales que se genera en la educación, se retroalimenta con la didáctica de las ciencias para transformar la forma de enseñarla (Aduriz, 2005a). De allí surge un interés por averiguar los conceptos epistémicos respecto a la imagen de ciencias que manejan los estudiantes de grado noveno y ampliarlos desde la representación de algunas situaciones sociales e históricas que marcan la transformación de los humedales de Bogotá, mediante el diseño y aplicación de una unidad didáctica que incluye como actividad central un videojuego, para analizar a partir de las habilidades cognitivolinguísticas planteadas por Jorba (2000), si se da una aproximación a una visión más socio científica. Por lo anterior, que los resultados pretendan llegar a reconocer en el lenguaje escrito y sustentado por el lenguaje oral de los educandos, nuevos valores epistémicos de la concepción humedal y allí se identifican razones significativas al hacerles ver que las nuevas ideas están 'justificadas' por la realidad tomada desde diversas percepciones, idea apoyada por De Longhi (2007).

B

I

0

0

N

E

N

\section{ABSTRACT}

The nature of science (NOS) is a metascience that articulates epistemology, history and sociology of science to contribute to the reflection on the image of science from social issues generated in education, is complemented with the didactic of science to transform the way of teaching (Aduriz, 2005a). From there comes an interest for finding out the epistemic concepts regarding the image of science that 9th graders have, and improve them from the representation of some social and historical situations that mark the transformation of wetlands in Bogotá, by designing and implementing a didactic unit which includes as core activity a videogame, to analyze from the cognitivelinguistic skills raised by Jorba (2000), if there is a socioscientific view. Therefore, the results intend to come to recognize in written language and supported by the oral language, new epistemic values of the wetland concept and there are we can find important reasons to make them see that new ideas are "justified " by reality taken from various perspectives, idea supported by De Longhi (2007).

${ }^{1}$ Universidad Distrital Francisco José de Caldas. susitaabella@gmail.com 
Edición Extraordinaria. p.p. $129-138$

Memorias del VIII Encuentro Nacional de Experiencias en Enseñanza de la Biología y la Educación Ambiental. III Congreso Nacional de Investigación en Enseñanza de la Biología.

PALABRAS CLAVE. Humedal, naturaleza de las ciencias, unidad didáctica, videojuego de rol.

KEYWORDS. Didactic unit, nature of science, role-playing game, wetlan.

\section{INTRODUCCIÓN}

Al contemplar la enseñanza de las ciencias, Porlán en (1997) señala que el propósito es ayudar a formar el hábito de cuestionar las ideas de los estudiantes y desarrollar estrategias adecuadas para contestarlas. Así mismo Sanmartí (2000a) habla de diversificar las actividades para generar motivación en los estudiantes, como por ejemplo incluir en las unidades didácticas los juegos y las dramatizaciones. Estas diversas actividades desarrollan habilidades que en muchas ocasiones son pasadas por alto por los docentes, conduciendo a pensar la ciencia de una manera tradicionalista y excluyendo imágenes de esta, que contemplan enfoques sociales, históricos y filosóficos, por ende es necesario replantear la imagen de ciencia que se muestra a los estudiantes. Para esta investigación, se retoma el desarrollo de las habilidades descriptivas y explicativas sugeridas por Jorba (2000) para favorecer los procesos de enseñanzaaprendizaje de la imagen de ciencia, en virtud de que aporta a la producción intelectual de los estudiantes, que debe hacer parte de la cultura ciudadana según Aduriz (2005a), Para representar situaciones históricas que han contribuido al cambio de los humedales desde el punto de vista diacrónico, "que aborde acontecimientos del pasado en términos del contexto vigente en la época considerada", (Lombardi, 1997, p.345) y aportar con el desarrollo cognitivo-linguístico, se recurre al diseño e implementación de un videojuego por ser potente promotor de aprendizaje. Por tanto se espera con el desarrollo de esta investigación cualitativa, aportar a la didáctica de las ciencias utilizando el videojuego como mediador, entre imagen de ciencia y una perspectiva social de los humedales.

La anterior idea es apoyada por Aduriz quien considera que:

La NOS agrupa saberes que sirven a modo de herramientas intelectuales para poder pensar sobre las ciencias en el ámbito de la formación de personas científicamente cultas, pero esos saberes han sufrido un proceso de selección y de transformación adaptativa desde su formulación originaria en el espacio académico de las metaciencias. (Aduriz, 2005, p.113). Adicionalmente McComas (1998) habla de la relevancia de NOS para estudiar la imagen de ciencia en los estudiantes, en los profesores y en otras poblaciones ya que se busca alejar la imagen distorsionada y hacer cada vez más familiar, esa imagen anhelada por quienes buscan enseñar una nueva ciencia. Por ende el objetivo general de esta investigación, busca estudiar la imagen de ciencia que se genera en estudiantes de grado noveno, cuando trabajan con una unidad didáctica mediada por un videojuego basado en sucesos históricos, sociales y ambientales de un humedal de Bogotá. 
Edición Extraordinaria. p.p. $129-138$

Memorias del VIII Encuentro Nacional de Experiencias en Enseñanza de la Biología y la Educación Ambiental. III Congreso Nacional de Investigación en Enseñanza de la Biología.

\section{METODOLOGÍA}

se integran aspectos como la Naturaleza de las Ciencias y la imagen de ciencia desde cuestiones históricas y sociales para tratar el concepto de humedal, las habilidades cognitivo-lingüísticas para reconocer la imagen de ciencia y el uso de los videojuegos en la educación en ciencias naturales. Este trabajo se enmarca dentro de la investigación cualitativa ya que responde a diferentes comportamientos y acciones frente a la cuestión sociocientífica que denominamos humedal. Es un estudio de caso y se colecta información escrita para analizar la producción individual de los estudiantes. Además la propuesta tiene un enfoque desde el paradigma socio-crítico de García, Llamas (2012), ellos plantean como principios: "conocer y comprender la realidad como praxis, unir teoría y práctica, orientar el conocimiento para liberar al individuo, implicar al docente a partir de la autorreflexión, y está encaminada a transformar la realidad, además de describirla y comprenderla".

Desde lo metodológico, las fases de implementación de la unidad didáctica se basan en Sanmartí (2000). Las actividades contempladas allí, son: un cuestionario para imagen de ciencia (tipo Likert) que consta de dos grandes categorías que corresponden a imagen tradicional de ciencia y a imagen avanzada, son 36 preguntas de las cuales 14 son tomadas de Aduriz (2001), e incluyen las categorías de relación teorías-hechos, naturaleza del conocimiento científico y su desarrollo, y las otras 22 son redactadas a partir de una adaptación a los tres enfoques que García (2004) propone para la educación ambiental. El autor menciona tres tendencias que hay sobre los paradigmas de la educación ambiental, el naturalista, el ambientalista y el de desarrollo sostenible que es para el cambio social. Otro instrumento es el videojuego en donde a través de cada uno de los escenarios diseñados, se relatan situaciones históricas ocurridas en torno a los humedales, como por ejemplo la urbanización, la percepción cultural del agua y el campo de acción científico de acuerdo a las necesidades sociales. El videojuego presenta distintos roles de manera neutra para que el estudiante piense de diferentes maneras el humedal, un concepto que es de la ciencia y la sociedad. La triangulación es metodológica, ya que se analizan los resultados del cuestionario para imagen de ciencia (tipo Likert) y las respuestas escritas sobre las misiones del videojuego.

En principio, para la fundamentación teórica se recurre a los estándares curriculares del M.E.N (2004), buscando que la planeación de las actividades didácticas se encuentren acordes con el grado escolar de la población, ya que las temáticas de biología culminan en grado noveno y por ello se eligen cinco estudiantes de este curso. Una vez seleccionada la población se realiza un tesauro con los términos claves como NOS, humedales, didáctica de las ciencias, imagen de ciencia, cuestiones sociocientíficas, videojuegos en la educación y EA. Acto seguido se afianzan los documentos necesarios para estado del arte y marco teórico, y se definen las categorías. Ahora la búsqueda 
Edición Extraordinaria. p.p. $129-138$

Memorias del VIII Encuentro Nacional de Experiencias en Enseñanza de la Biología y la Educación Ambiental. III Congreso Nacional de Investigación en Enseñanza de la Biología.

para elegir los momentos de la historia de los humedales de Bogotá que se apoyan en el videojuego.

Para el diseño de las actividades de la unidad didáctica, se seleccionan siguiendo la clasificación dada por Sanmartí (2000), que consta de 5 fases como ya se mencionó.

- Exploración: se aplica un instrumento para imagen de ciencia (tipo Likert) de 36 preguntas. Todas las preguntas con el fin de conocer si su imagen de ciencia es tradicional o avanzada de acuerdo a las preguntas que van en consonancia con las categorías. Luego por medio de un mapa conceptual, cada estudiante expone lo que sabe acerca del tema humedal. (Es necesario resaltar que previamente se trabaja en la elaboración de mapas conceptuales)

- Introducción de nuevos puntos de vista: por medio de una guía de trabajo y una salida al humedal santa María del lago, los estudiantes deben comenzar a evidenciar situaciones naturales, luego antropocéntricas y culturales. El videojuego también se incluye en esta categoría.

- Síntesis: a través del videojuego y las sesiones de aplicación se presenta información sobre sucesos que modificaron las características de los humedales. Por medio del chat en línea del juego, se generan cuestionamientos para dirigir las sesiones y orientar a los estudiantes, de manera que no se dejen solos los procesos de aprendizaje.

- Aplicación: los nuevos conocimientos y visiones se reflejan mediante escritos sobre humedales y un nuevo mapa conceptual en el que pueden incluir ideas, descripciones, conceptos y explicaciones entre otras.

Generalización: los estudiantes reconocen si su forma de pensar (en la ciencia y la relación de esta con lo ambiental y por consiguiente con los humedales) se mantiene al auto revisar su instrumento para imagen de ciencia (tipo Likert) inicial o si se dan variaciones en lo que quieren responder al finalizar toda la unidad didáctica.

Posteriormente en la fase de diseño del videojuego, este es elaborado pensando en brindarle a los estudiantes diferentes persepctivas de la ciencia desde los humedales y por ello cada escenario refleja contextos de ciencia desde direfentes roles sociales.

Además de las sesiones de videojuego se utilizan actividades complementarias como la elaboración de escritos, de mapas conceptuales, la revisión de algunos conceptos nuevos, una salida de campo y algunas explicaciones complementarias que surgían sobre la marcha. Toda la información es colectada mediante tablas con preguntas abiertas, el chat del videojuego y escritos libres (cada uno de máximo tres párrafos). El material producido por cada estudiante es enviado dentro de cada sesión, al correo de la docente, de manera que la mayoría de información es obtenida de forma digital. 
Edición Extraordinaria. p.p. $129-138$

Memorias del VIII Encuentro Nacional de Experiencias en Enseñanza de la Biología y la Educación Ambiental. III Congreso Nacional de Investigación en Enseñanza de la Biología.

En el siguiente diagrama se presentan las diferentes fases metodológicas desarrolladas a lo largo de la investigación.

Diagrama 1. Metodología de la investigación

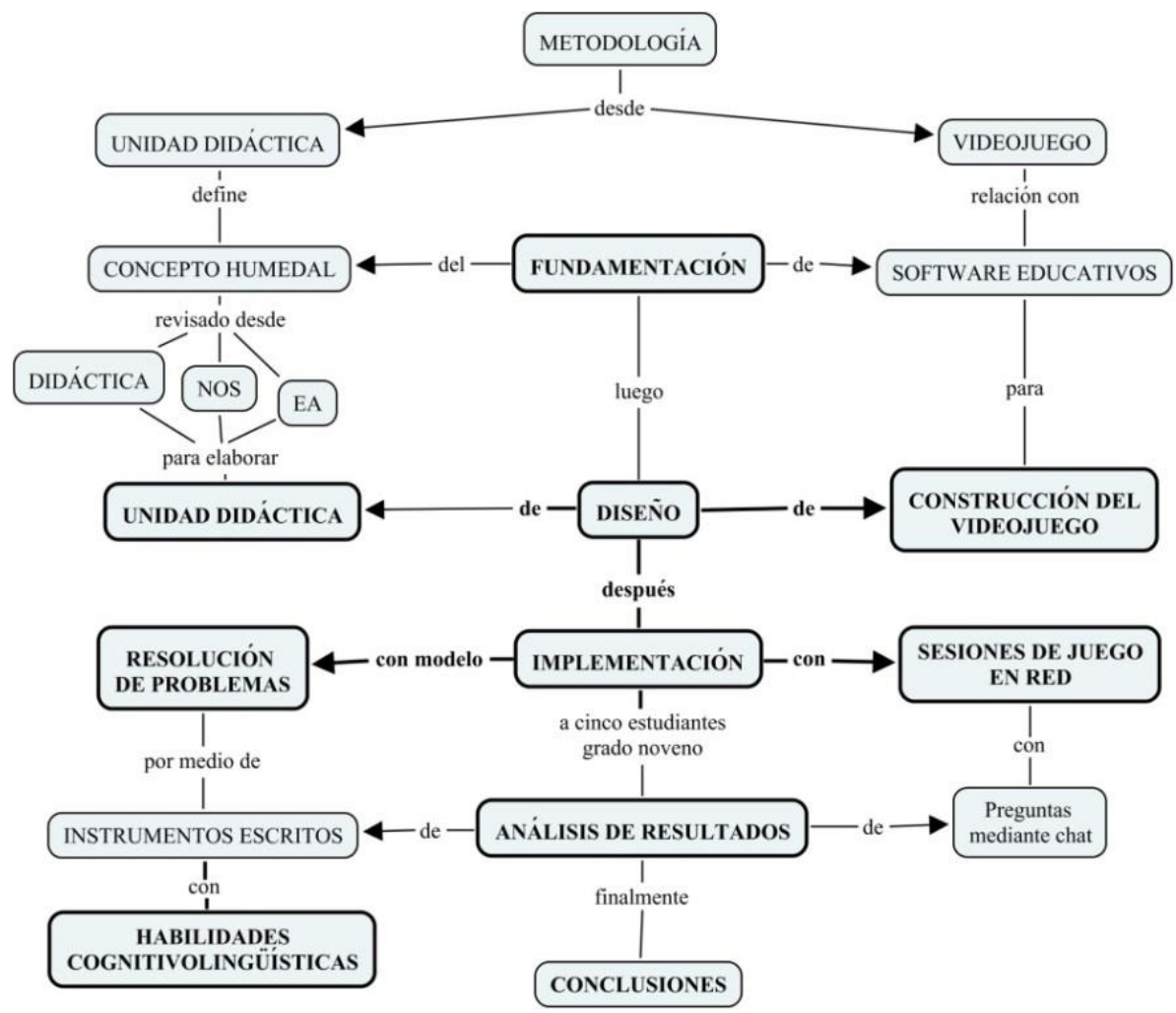

El videojuego refleja dos grandes mundos que son una representación de los humedales en general, pero que está basado en el humedal Santa María del Lago. Uno de los mundos presenta ciertas características de los humedales en el año de 1.930 y el otro corresponde a 2014. En seguida se muestra la tabla que relaciona las categorías del trabajo de investigación, con la justificación de cada misión y las preguntas que se abordan para cada uno de los escenarios.

Tabla 1. Relación videojuego y categorías de análisis

\begin{tabular}{|c|c|c|c|c|}
\hline \multirow[t]{2}{*}{$\begin{array}{c}\text { SUB } \\
\text { CATEGORIA }\end{array}$} & \multirow[t]{2}{*}{ SE RETOMA PORQUE } & \multirow[t]{2}{*}{ MISIÓN } & \multirow[t]{2}{*}{ LO QUE SE BUSCA } & $\begin{array}{l}\text { PREGUNTAS EN EL } \\
\text { JUEGO }\end{array}$ \\
\hline & & & & \begin{tabular}{|l|l|}
1930 & 2014 \\
\end{tabular} \\
\hline $\begin{array}{l}\text { SMA } \\
\text { Sensibilización } \\
\text { medio } \\
\text { ambiente }\end{array}$ & $\begin{array}{l}\text { Señala aspectos } \\
\text { importantes como lo } \\
\text { cultural y algo histórico } \\
\text { por la apropiación de } \\
\text { diferentes espacios no } \\
\text { solamente para ser } \\
\text { explotados, sino por la } \\
\text { responsabilidad de ser }\end{array}$ & Indígena & $\begin{array}{l}\text { Que se evidencie la diferencia entre } \\
\text { el estado del cuerpo de agua } \\
\text { puesto que hace muchos años se } \\
\text { podía pescar allí, pero ahora es una } \\
\text { necesidad recuperar estos espacios } \\
\text { al procurar mantenerlos limpios. } \\
\text { Por otra parte, hay una perspectiva } \\
\text { cultural frente a los Muiscas que }\end{array}$ & $\begin{array}{l}\text { - Describe el mundo SIE. } \\
\text { - ¿Qué usos se hace del } \\
\text { agua en este mundo? Ten } \\
\text { en cuenta la labor de Cintya }\end{array}$ \\
\hline
\end{tabular}


Edición Extraordinaria. p.p. $129-138$
Memorias del VIII Encuentro Nacional de Experiencias en Enseñanza de la Biología y la Educación Ambiental. III Congreso Nacional de Investigación en Enseñanza de la Biología.

\begin{tabular}{|c|c|c|c|c|c|}
\hline \multirow[t]{2}{*}{$\begin{array}{c}\text { SUB } \\
\text { CATEGORIA }\end{array}$} & \multirow[t]{2}{*}{ SE RETOMA PORQUE } & \multirow[t]{2}{*}{ MISIÓN } & \multirow[t]{2}{*}{ LO QUE SE BUSCA } & \multicolumn{2}{|c|}{$\begin{array}{c}\text { PREGUNTAS EN EL } \\
\text { JUEGO }\end{array}$} \\
\hline & & & & 1930 & 2014 \\
\hline & preservados. & & $\begin{array}{l}\text { buscaban habitar lugares cercanos } \\
\text { al agua pero estas tradiciones se } \\
\text { han ido perdiendo por los cambios } \\
\text { que allí (en los humedales) han } \\
\text { ocurrido. }\end{array}$ & & \\
\hline $\begin{array}{l}\text { MSA } \\
\text { Mirada socio } \\
\text { ambiental }\end{array}$ & $\begin{array}{l}\text { Al involucrar al civil en } \\
\text { actividades que } \\
\text { relacionan el humedal } \\
\text { con lo económico y } \\
\text { social, se contemplan } \\
\text { otras perspectivas que } \\
\text { demuestran que no se } \\
\text { pueden desligar las } \\
\text { problemáticas } \\
\text { ambientales de los } \\
\text { roles mencionados. }\end{array}$ & Civil & $\begin{array}{l}\text { Es importante reconocer que } \\
\text { aunque se han perdido varias } \\
\text { hectáreas de zonas verdes y un } \\
\text { cuerpo de agua completo, se } \\
\text { realizan algunas actividades para } \\
\text { delimitar los espacios antrópicos y } \\
\text { naturales. Además, comprender } \\
\text { que el hecho de que las funciones } \\
\text { del suelo cambiaron y estos } \\
\text { entornos no son rurales, no significa } \\
\text { que sea negativo, sino que los } \\
\text { cultivos se han ido desplazando a } \\
\text { lugares menos urbanizados a las } \\
\text { afueras de la ciudad y que aún se } \\
\text { mantienen. }\end{array}$ & $\begin{array}{l}\text { ¿Qué } \\
\text { piensas } \\
\text { de la } \\
\text { siembra } \\
\text { en estos } \\
\text { terrenos? } \\
\text { Es buena } \\
\text { o mala y } \\
\text { ¿por } \\
\text { qué? }\end{array}$ & $\begin{array}{l}\text { ¿Crees que } \\
\text { los jarillones } \\
\text { benefician al } \\
\text { hombre y al } \\
\text { humedal? } \\
\text { ¿Por qué? }\end{array}$ \\
\hline $\begin{array}{l}\text { RAyE } \\
\text { Relación } \\
\text { ambiente y } \\
\text { entorno } \\
\text { NCC } \\
\text { Naturaleza del } \\
\text { Conocimiento } \\
\text { Científico. }\end{array}$ & $\begin{array}{l}\text { Los dos escenarios } \\
\text { permiten ver que la } \\
\text { ciencia va cambiando } \\
\text { en su accionar, de } \\
\text { acuerdo a las } \\
\text { necesidades que } \\
\text { surgen para el hombre } \\
\text { y su medio. Cuando se } \\
\text { reduce un concepto a } \\
\text { lo ecológico se sesgan } \\
\text { vínculos que existen } \\
\text { desde otros contextos. } \\
\text { Además, la ciencia da } \\
\text { sustento teórico para } \\
\text { plantear soluciones a } \\
\text { problemas que se } \\
\text { resuelven no } \\
\text { solamente por un } \\
\text { científico, evidenciado } \\
\text { la construcción de la } \\
\text { ciencia colectiva. }\end{array}$ & Científico & $\begin{array}{l}\text { La labor del científico es diferente } \\
\text { aunque igualmente importante en } \\
\text { ambos escenarios, por ello es } \\
\text { crucial que el estudiante identifique } \\
\text { que desde una visión tradicional, } \\
\text { sobresalían actividades de } \\
\text { descripción basadas en la } \\
\text { observación. Posteriormente } \\
\text { diferentes situaciones de ciencia se } \\
\text { hacen cercanas a todos y se busca } \\
\text { ser participativo, ayudando a la } \\
\text { recuperación. Se busca también } \\
\text { que los estudiantes identifiquen la } \\
\text { importancia de conocer lo teórico } \\
\text { para reconocer un problema y } \\
\text { actuar en consecuencia. }\end{array}$ & $\begin{array}{l}\text { ¿Qué b } \\
\text { labor } \\
\text { De Log } \\
\text { Pluma } \\
\text { qué? }\end{array}$ & $\begin{array}{l}\text { neficios trae la } \\
\text { personaje? } \\
\text { cio en } 1930 \text { y } \\
2014 \text { ¿Por }\end{array}$ \\
\hline
\end{tabular}


Edición Extraordinaria. p.p. $129-138$

Memorias del VIII Encuentro Nacional de Experiencias en Enseñanza de la Biología y la Educación Ambiental. III Congreso Nacional de Investigación en Enseñanza de la Biología.

\begin{tabular}{|c|c|c|c|c|c|}
\hline \multirow[t]{2}{*}{$\begin{array}{c}\text { SUB } \\
\text { CATEGORIA }\end{array}$} & \multirow[t]{2}{*}{ SE RETOMA PORQUE } & \multirow[t]{2}{*}{ MISIÓN } & \multirow[t]{2}{*}{ LO QUE SE BUSCA } & \multicolumn{2}{|c|}{$\begin{array}{c}\text { PREGUNTAS EN EL } \\
\text { JUEGO }\end{array}$} \\
\hline & & & & 1930 & 2014 \\
\hline $\begin{array}{l}\text { MSA } \\
\text { (Mirada socio } \\
\text { ambiental) } \\
\text { RAyE } \\
\text { (Relación } \\
\text { ambiente } \\
\text { entorno). }\end{array}$ & $\begin{array}{l}\text { Amplia perspectivas de } \\
\text { cuestiones sociales, } \\
\text { con diferentes posturas } \\
\text { y roles. } \\
\text { Se pueden evidenciar } \\
\text { las consideraciones } \\
\text { frente a las acciones de } \\
\text { la dualidad } \\
\text { hombre/ambiente. }\end{array}$ & Urbanizador & $\begin{array}{l}\text { Analizar estas situaciones permite } \\
\text { evidenciar que la urbanización ha } \\
\text { cambiado la forma de vivir. } \\
\text { Generalmente comparaciones como } \\
\text { estas caen en críticas como si se } \\
\text { quisiera vivir ruralmente, sin } \\
\text { embargo al ver los beneficios que } \\
\text { trae lo urbano y la búsqueda del } \\
\text { desarrollo de la ciudad, se } \\
\text { evidencian más comodidades y } \\
\text { facilidades de transporte, } \\
\text { alimentación, trabajo y educación. }\end{array}$ & $\begin{array}{l}\text { ¿Te gustó } \\
\text { que el } \\
\text { premio } \\
\text { fuera una } \\
\text { casa en } \\
\text { este } \\
\text { lugar? } \\
\text { ¿Por qué }\end{array}$ & $\begin{array}{lr}\text { ¿Te gustó } \\
\text { que el } \\
\text { premio que } \\
\text { te dieron en } \\
\text { esta misión } \\
\text { fuera un } \\
\text { apartamento } \\
\text { ? ¿Por qué? }\end{array}$ \\
\hline $\begin{array}{l}\text { MSA } \\
\text { (Mirada socio } \\
\text { ambiental) }\end{array}$ & $\begin{array}{l}\text { Involucra uno de los } \\
\text { principales ejes que se } \\
\text { relaciona con las } \\
\text { decisiones ambientales } \\
\text { y sociales, en torno a la } \\
\text { temática humedal }\end{array}$ & Político & $\begin{array}{l}\text { Inicialmente, se pretende mostrar al } \\
\text { político como un funcionario que } \\
\text { busca mejorar la calidad de vida de } \\
\text { los diferentes habitantes del lugar y } \\
\text { para ello necesita de permisos para } \\
\text { construir y crecer aún más. No se } \\
\text { quiere estigmatizar ninguna de las } \\
\text { perspectivas que retoma el } \\
\text { videojuego por eso es importante } \\
\text { que desde una visión amplia, los } \\
\text { estudiantes comprendan las } \\
\text { razones del otro. Posteriormente, se } \\
\text { evidencian cambios que han } \\
\text { ocurrido realmente, El ultimo } \\
\text { escenario es más ficticio y y } \\
\text { apocalíptico, solo es una posibilidad } \\
\text { exagerada para que se evidencie } \\
\text { que entre todos se construye } \\
\text { sociedad y que el humedal (como } \\
\text { situación social que se trabaja } \\
\text { desde la ciencia), es un pequeño } \\
\text { ejemplo de complejidad y varias } \\
\text { perspectivas que favorecen y } \\
\text { desfavorecen dependiente desde } \\
\text { donde se analice. }\end{array}$ & $\begin{array}{l}\text { ¿Cuál será } \\
\text { el futuro de } \\
\text { este } \\
\text { mundo? } \\
\text { Los papeles } \\
\text { que } \\
\text { entregaste } \\
\text { ayudan a } \\
\text { crecer y } \\
\text { embellecer } \\
\text { la ciudad ya } \\
\text { que eran } \\
\text { permisos } \\
\text { para } \\
\text { continuar } \\
\text { proyectos. } \\
\text { ¿Crees que } \\
\text { eso está } \\
\text { bien o mal y } \\
\text { por qué? }\end{array}$ & $\begin{array}{l}\text { Asume uno } \\
\text { de los } \\
\text { personajes } \\
\text { que dan } \\
\text { misiones y } \\
\text { cuenta que } \\
\text { habrías } \\
\text { hecho de ser } \\
\text { él o ella. } \\
\text { (Bien sea en } \\
\text { 1930 o en } \\
2014 \text { ) }\end{array}$ \\
\hline
\end{tabular}

\section{RESULTADOS Y DISCUSIÓN}

Uno de los resultados principales es el videojuego finalizado, siendo este pensado desde la imagen de ciencia a partir de los humedales. A continuación se presenta uno de los escenarios para que el lector se haga una idea de la estructura del videojuego y de las situaciones allí planteadas que sirvieron para generar las respuestas de los estudiantes. 
Edición Extraordinaria. p.p. $129-138$

Memorias del VIII Encuentro Nacional de Experiencias en Enseñanza de la Biología y la Educación Ambiental. III Congreso Nacional de Investigación en Enseñanza de la Biología.

Tabla 2. Videojuego con misiones.

\begin{tabular}{|c|c|c|c|}
\hline \multicolumn{2}{|c|}{ MISIÓN DEL VIDEOJUEGO } & \multicolumn{2}{|c|}{ IMAGEN HIDRATA } \\
\hline 1930 & 2014 & PERSONAJE & E = CIENTÍFICO \\
\hline $\begin{array}{l}\text { Se encuentra } \\
\text { observado las } \\
\text { diferentes especies y } \\
\text { tomando notas de } \\
\text { tipo etológico, pide } \\
\text { que se le ayude a } \\
\text { recuperar un libro de } \\
\text { apuntes que le han } \\
\text { robado en donde } \\
\text { dibujaba diferentes } \\
\text { ejemplares de los } \\
\text { que contemplaba en } \\
\text { aquel lugar }\end{array}$ & $\begin{array}{l}\text { Al observar la proliferación } \\
\text { del buchón de agua, su } \\
\text { trabajo consiste en retirar } \\
\text { estos vegetales y para ello } \\
\text { pide ayuda, puesto que se } \\
\text { quedan en la superficie y } \\
\text { no permiten que se } \\
\text { desarrolle el ciclo } \\
\text { hidrológico de manera } \\
\text { normal. Además } \\
\text { consumen oxigeno } \\
\text { necesario para otras } \\
\text { especies que se } \\
\text { encuentran allí. }\end{array}$ & 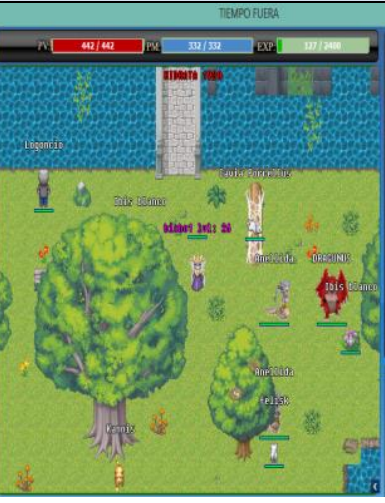 & 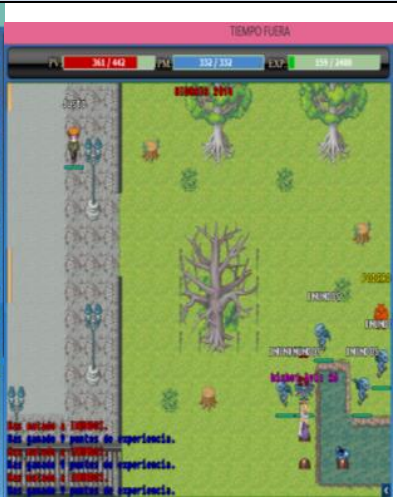 \\
\hline
\end{tabular}

En la siguiente tabla se observa un ejemplo del tratamiento que se dio a los textos generados por los estudiantes para la sistematización y análisis en donde se contrastan respuestas iniciales, intermedias y finales.

Tabla 3. Tabla de correlación para análisis

\begin{tabular}{|c|c|c|c|c|c|}
\hline Estudiante & Escrito & Análisis & $\begin{array}{c}\text { Imagen de } \\
\text { Ciencia }\end{array}$ & $\begin{array}{c}\text { Habilidad } \\
\text { cognitivolingüística }\end{array}$ & Resumen \\
\hline & & & & & \\
\hline
\end{tabular}

\section{CONCLUSIONES}

Con el material descrito anteriormente, se analiza que en términos generales la categoría de relación teorías-hechos no expresa cambios significativos en los estudiantes y esto se debe a que no era un tema explicito dentro de las misiones y se buscaba que a partir de situaciones científicas ellos reinterpretaran la construcción de la ciencia. Para la naturaleza del conocimiento científico y el desarrollo del mismo, hay más cambios hacia imagen avanzada pues el hecho de interactuar con un científico en 
Memorias del VIII Encuentro Nacional de Experiencias en Enseñanza de la Biología y la Educación Ambiental. III Congreso Nacional de Investigación en Enseñanza de la Biología.

dos épocas diferentes y comparar el quehacer, permite comprender que no basta con describir y explicar lo que ocurre en la naturaleza, sino que el mundo necesita de diferentes métodos de investigación y vincular estas con necesidades sociales. En la categoría ambiental que incluye disciplinar-mecanicista, sensibilización ambiental, relación ambiente-entorno y mirada socio-ambiental, el videojuego evidencia la efectividad de las misiones que se planearon pensadas desde lo cultural, político, social, económico e histórico que no muestran como enemigo a ninguno de estos contextos, sino que por el contrario busca mostrar que la ciencia se relaciona con lo ambiental y que directa o indirectamente todos hacen parte de ella y que más allá de saber lo netamente teórico, se necesita la aplicación para actuar en aras de una reflexión epistemológica.

Por otra parte, las categorías de naturaleza del conocimiento científico, desarrollo del conocimiento científico y las correspondientes a la perspectiva ambiental, se movilizan de tradicional a avanzada por retomar factores lógicos y sociales como inherentes a la construcción de las ciencias.

\section{BIBLIOGRAFÍA}

Aduriz, A. (2001), Integración de la epistemología en la formación del profesorado de ciencias, (tesis doctoral). Universitat Autònoma de Barcelona Departament de Didàctica de les Matemàtiques i de les Ciències Experimentals. Barcelona España.

Aduriz, A. (2005a). Una introducción a la naturaleza de las ciencias, la epistemología en la enseñanza de las ciencias naturales. El salvador, Buenos Aires Argentina. Fondo de cultura y economía S.A.

De longhi. A., Campaner. G. (2007) la argumentación en educación ambiental. Una estrategia didáctica para la escuela media. Universidad nacional de córdoba Argentina, pp. (443445) recuperado de http://www.saum.uvigo.es/reec/volumenes/volumen6/art12_vol6_n2.pdf

García, E. (2004). Educación Ambiental, Constructivismo y Complejidad. Diada editora, serie fundamentos número 20, colección investigación y enseñanza. Sevilla España.

García, Llamas, (2012). Métodos de investigación en educación (pp 30-47). Madrid: UNED

Jorba, J, Gómez, I, \& Prat, À. (2000). Hablar y escribir para aprender: Uso de la lengua en situación de enseñanza-aprendizaje desde las áreas curriculares. Madrid: Síntesis/ICE de la UAB

Lombardi. O, (1997). La pertinencia de la historia en la enseñanza de las ciencias: argumentos y contraargumentos. Gabinete de Desarrollo de Metodologías de la Enseñanza. Facultad de Ingeniería. Universidad de Buenos Aires. Av. Paseo de Colón, 850,2". 1063 Buenos Aires. Argentina.

McComas, W. (1998). The nature of science in science education. Rationales and strategies. Dordrecht: Kluwer. 
Bio - grafía. Escritos sobre la Biología y su Enseñanza. ISSN 2027-1034

Edición Extraordinaria. p.p. $129-138$

Memorias del VIII Encuentro Nacional de Experiencias en Enseñanza de la Biología y la

Educación Ambiental. III Congreso Nacional de Investigación en Enseñanza de la Biología.

M.E.N. (2004), Revolución Educativa Colombia Aprende. Santa Fe de Bogotá. Ministerio de Educación Nacional.

Porlán, R. Rivero, A. Del Pozo, R. (1997). Conocimiento profesional y epistemología de los profesores I. teoría, métodos e instrumentos. Enseñanza de las ciencias: revista de investigación y experiencias didácticas, ISSN 0212-4521 , Vol. 15, N² 2, 1997, págs. $155-172$

Sanmartí, N. (2000). El diseño de unidades didácticas. Didáctica de las ciencias experimentales: teoría y práctica de la enseñanza de las ciencias / coord. por Francisco Javier Perales Palacios, Pedro Cañal de León, 2000, ISBN 84-268-1051-9, págs. 239. 